\title{
Runge-Kutta Method and Bolck by Block Method to Solve Nonlinear Fredholm-Volterra Integral Equation with Continuous Kernel
}

\author{
A. M. Al-Bugami, J. G. Al-Juaid \\ Department of Mathematics, Faculty of Sciences, Taif University, Taif, KSA \\ Email: abeer101aa@yahoo.com
}

How to cite this paper: Al-Bugami, A.M. and Al-Juaid, J.G. (2020) Runge-Kutta Method and Bolck by Block Method to Solve Nonlinear Fredholm-Volterra Integral Equation with Continuous Kernel. Journal of Applied Mathematics and Physics, 8, 20432054.

https://doi.org/10.4236/jamp.2020.89152

Received: August 10, 2020

Accepted: September 27, 2020

Published: September 30, 2020

Copyright $\odot 2020$ by author(s) and Scientific Research Publishing Inc. This work is licensed under the Creative Commons Attribution International License (CC BY 4.0).

http://creativecommons.org/licenses/by/4.0/

\begin{abstract}
In this paper, the existence and uniqueness of the solution of Fredholm-Volterra integral equation is considered (NF-VIE) with continuous kernel; then we used a numerical method to reduce this type of equations to a system of nonlinear Volterra integral equations. Runge-Kutta method (RKM) and Bolck by block method (BBM) are used to solve the system of nonlinear Volterra integral equations of the second kind (SNVIEs) with continuous kernel. The error in each case is calculated.
\end{abstract}

\section{Keywords}

Nonlinear Fredholm-Volterra Integral Equation, System of Nonlinear Volterra Integral Equations, Runge-Kutta Method, Bolck by Block Method

\section{Introduction}

Integral equations of various types and kinds play an important role in many branches of linear and nonlinear function analysis and their applications in the theory of elasticity, engineering, mathematical physical and contact mixed problems. Therefor, many different methods are used to obtain the solution of the Volterra integral equation. In [1] Linz, studied analytical and numerical methods of Volterra equation. In [2], Mirzaee and Rafei used the BBM for the numerical solution of the nonlinear two-dimensional Volterra integral equations. In the references [3]-[8] the authors considered many different methods to solve linear and nonlinear system of Volterra integral equations numerically with continuous and singular kernels. In [9], Al-waqdani studied linear F-VIE with continuous kernel and solved the linear SVIEs numerically with continuous kernel. 
$\mu \phi(x, t)=f(x, t)+\lambda \int_{a}^{b} K(x, y) \gamma(y, t, \phi(y, t)) \mathrm{d} y+\lambda \int_{0}^{t} F(t, \tau) \gamma(x, \tau, \phi(x, \tau)) \mathrm{d} \tau$

Equation (1) is called the NF-VIE in the space $L_{2}[a, b] \times C[0, T], T<\infty$. Here the Fredholm integral term is considered in position with a positive continuous kernel $K(x, y)$ for all $x, y \in[a, b]$, while the Volterra integral term is considered in time with a positive continuous kernel $F(t, \tau)$ for all $t, \tau \in[0, T], T<\infty$. The free term $f(x, t)$ is known continuous function in the space $L_{2}[a, b] \times C[0, T]$, while $\phi(x, t)$ is unknown function representing the solution of the nonlinear integral Equation (1). The numerical coefficient $\lambda$ is called the parameter of the integral equation, may be complex, and has physical meaning, while the constant parameter $\mu$ defines the kind of the integral Equation (1).

\section{Existence of Solution of NF-VIE}

To prove the existence of a unique solution of Equation (1) using fixed point theorem.

We write it in the integral operator form:

$$
\bar{W} \phi=f+W \phi
$$

where

$$
\begin{gathered}
W \phi=K \phi+F \phi \\
K \phi=\lambda \int_{a}^{b} K(x, y) \gamma(y, t, \phi(y, t)) \mathrm{d} y, F \phi=\lambda \int_{0}^{t} F(t, \tau) \gamma(x, \tau, \phi(x, \tau)) \mathrm{d} \tau
\end{gathered}
$$

Then, we assume the following conditions:

i) The kernel of Fredholm integral term satisfies:

$$
|K(x, y)| \leq A_{1} \quad\left(A_{1} \text { is a constant }\right) .
$$

ii) The kernel of Volterra integral term satisfies:

$$
|F(t, \tau)| \leq A_{2} \quad\left(A_{2} \text { is a constant }\right) .
$$

iii) The given function $f(x, t)$ with its partial derivatives is continuous in $L_{2}[a, b] \times C[0, T]$ where:

$$
\|f(x, t)\|=\max \int_{0}^{t}\left(\int_{a}^{b}|f(x, \tau)|^{2} \mathrm{~d} x\right)^{\frac{1}{2}} \mathrm{~d} \tau=A_{3} \quad\left(A_{3} \quad \text { is a constant }\right) .
$$

iv) The known continuous function $\gamma(x, t, \phi(x, t))$, for the constant $B>B_{1}, B>p$, the following conditions:
a) $\left(\int_{0}^{t} \int_{a}^{b}|\gamma(x, t, \phi(x, t))|^{2} \mathrm{~d} x \mathrm{~d} t\right)^{\frac{1}{2}} \leq B_{1}\|\phi(x, t)\|_{L_{2}[a, b] \times C[0, T]}$
b) $\left\|\gamma\left(x, t, \phi_{1}(x, t)\right)-\gamma\left(x, t, \phi_{2}(x, t)\right)\right\| \leq N(x, t)\left|\phi_{1}(x, t)-\phi_{2}(x, t)\right|$ where $\|N(x, t)\|_{L_{2}[a, b] \times C[0, T]}=p$.

Theorem 1:

If the condition i)-iv) are verified, then Equation (1) has unique solution in the Banach space $L_{2}[a, b] \times C[0, T], T<\infty$. 
The provement of this theorem depends on the following two lemmas:

\section{Lemma 1:}

Under the conditions i)-iv-a), the operator $\bar{W}$ defined by (2), maps the space $L_{2}[a, b] \times C[0, T], T<\infty$ into itself.

Proof:

In view of Formula (2) and (3) we get:

$$
\begin{aligned}
\|\bar{W} \phi(x, t)\| \leq & \|f(x, t)\|+|\lambda|\left\|\int_{a}^{b}|K(x, y)\|\gamma(y, t, \phi(y, t)) \mid \mathrm{d} y\|\right. \\
& +|\lambda|\left\|\int_{0}^{t}|F(t, \tau)\|\gamma(x, \tau, \phi(x, \tau)) \mid \mathrm{d} \tau\|\right.
\end{aligned}
$$

Using the conditions (i)-(iii), then applying Cauchy-Schwarz inequality, we have:

$$
\|\bar{W} \phi(x, t)\| \leq A_{3}+|\lambda| A_{1}\left(\int_{a}^{b}|\gamma(y, t, \phi(y, t))|^{2} \mathrm{~d} y\right)^{\frac{1}{2}}
$$

$$
+|\lambda| A_{2}\left(\int_{0}^{t}|\gamma(x, \tau, \phi(x, \tau))|^{2} \mathrm{~d} \tau\right)^{\frac{1}{2}}
$$

In the light of the condition (iv-a), the above inequality take the form:

$$
\|\bar{W} \phi(x, t)\| \leq A_{3}+\alpha\|\phi(x, t)\|, \quad \alpha=|\lambda| B_{1}\left(A_{1}+A_{2}+L\right)
$$

The lost inequality (5) shows that, the operator $\bar{W}$ maps the ball $S_{r}$ into itself, where

$$
r=\frac{A_{3}}{1-|\lambda| B_{1}\left(A_{1}+A_{2} L\right)}
$$

Since $r>0, A_{3}>0$, therefore we have $\alpha<1$. Moreover, the inequality (5) involves the boundedness of the operator $W$ of Equation (2) where:

$$
\|W \phi(x, t)\| \leq \alpha\|\phi(x, t)\|
$$

Also, the inequalities (5) and (7) define the boundedness of the operator $\bar{W}$.

Lemma 2:

If the conditions (i),(ii) and (iv-b) are satisfied, then the operator $\bar{W}$ is contractive in space $L_{2}[a, b] \times C[0, T], T<\infty$.

Proof:

For two functions $\phi_{1}(x, t)$ and $\phi_{2}(x, t)$ in the space $L_{2}[a, b] \times C[0, T]$ Formula (2), (3) leads to:

$$
\begin{aligned}
\left\|\bar{W} \phi_{1}(x, t)-\bar{W} \phi_{2}(x, t)\right\| \leq & |\lambda|\left\|\int _ { a } ^ { b } \left|K(x, y)\left\|\gamma\left(y, t, \phi_{1}(y, t)\right)-\gamma\left(y, t, \phi_{2}(y, t)\right) \mid \mathrm{d} y\right\|\right.\right. \\
& +|\lambda||| \int_{0}^{t}\left|F(t, \tau)\left\|\gamma\left(x, \tau, \phi_{1}(x, \tau)\right)-\gamma\left(x, \tau, \phi_{2}(x, \tau)\right) \mid \mathrm{d} \tau\right\|\right.
\end{aligned}
$$

Using the condition (iv-b), then apply Cauchy-Schwarz inequality we have:

$$
\begin{aligned}
\left\|\bar{W} \phi_{1}(x, t)-\bar{W} \phi_{2}(x, t)\right\| \leq & |\lambda||K(x, y)|\left(\int_{a}^{b} N^{2}(x, t)\left|\phi_{1}(y, t)-\phi_{2}(y, t)\right|^{2} \mathrm{~d} y\right)^{\frac{1}{2}} \\
& +|\lambda \|| F(t, \tau) \mid\left(\int_{a}^{b} N^{2}(x, \tau)\left|\phi_{1}(x, \tau)-\phi_{2}(x, \tau)\right|^{2} \mathrm{~d} \tau\right)^{\frac{1}{2}}
\end{aligned}
$$

Finally, with the aid of conditions (i), (ii), and (iv-b) we obtain: 


$$
\left\|\bar{W} \phi_{1}(x, t)-\bar{W} \phi_{2}(x, t)\right\| \leq \alpha\left\|\phi_{1}(x, t)-\phi_{2}(x, t)\right\|
$$

In equality (8) shows that, the operator $\bar{W}$ is continuous in the space $L_{2}[a, b] \times C[0, T], T<\infty$, then $\bar{W}$ is a contraction operator under the condition $\alpha<1$.

\section{The SVIES}

Consider:

$$
\begin{aligned}
\phi(x, t)= & f(x, t)+\lambda \int_{a}^{b} K(x, y) \gamma(y, t, \phi(y, t)) \mathrm{d} y \\
& +\lambda \int_{0}^{t} F(t, \tau) \gamma(x, \tau, \phi(x, \tau)) \mathrm{d} \tau,(\mu=1)
\end{aligned}
$$

when $a=b=0$, Equation (9) becomes:

$$
\phi(0, t)=f(0, t)+\lambda \int_{0}^{t} F(t, \tau) \gamma(0, \tau, \phi(0, \tau)) \mathrm{d} \tau
$$

Then,

$$
\phi_{0}(t)=f_{0}(t)+\lambda \int_{0}^{t} F(t, \tau) \gamma\left(\tau, \phi_{0}(\tau)\right) \mathrm{d} \tau
$$

where $u_{0}(t)=u(0, t), f_{0}(t)=f(0, t)$.

Formula (10) represents Volterra integral equation of the second kind at $a=b=0$. For representing (9) as a VIEs, we use the numerical method. Divide the interval $[a, b]$ as $a=x_{0} \leq x_{1} \leq \cdots \leq x_{N}=b$. Using the quadrature formula, Equation (9) becomes:

$$
\begin{aligned}
& \int_{a}^{b} K(x, y) \gamma(y, t, \phi(y, t)) \mathrm{d} y \\
& =\sum_{m=0}^{n} u_{m} K\left(x_{n}, x_{m}\right) \gamma\left(x_{n}, t, \phi\left(x_{n}, t\right)\right)
\end{aligned}
$$

where $n=1,2, \cdots, N$, and $u_{0}=\frac{1}{2} h_{0}, u_{n}=\frac{1}{2} h_{n}, u_{i}=h_{i},(i \neq 0, n)$.

Using (11) in (9), we have:

$$
\begin{aligned}
\phi(x, t)= & f(x, t)+\lambda \int_{0}^{t} F(t, \tau) \gamma(x, \tau, \phi(x, \tau)) \mathrm{d} \tau \\
& +\sum_{m=0}^{n} u_{m} K\left(x_{n}, x_{m}\right) \gamma\left(x_{n}, t, \phi\left(x_{n}, t\right)\right)
\end{aligned}
$$

Then:

$$
\phi_{n}=W_{n}(t)+\lambda \int_{0}^{t} F(t, \tau) \gamma\left(x_{n}, \tau, \phi_{n}(\tau)\right) \mathrm{d} \tau
$$

where $W_{n}(t)=f_{n}(t)+\lambda \sum_{m=0}^{n-1} u_{m} K\left(x_{n}, x_{m}\right) \gamma\left(x_{m}, t, \phi_{m}(t)\right), n=0,1, \cdots, N$. Formula (13) represents a NSVIEs of the second kind, and we have $N$ unknown functions $\phi_{n}(t)$ corresponding to time interval $[0, T]$. 


\section{Some Numerical Methods for Solving SVIEs}

\subsection{RKM}

In this section, the RKM is used to solve NF-VIE of the second kind. By divide the interval $[a, b]$ as $a=x_{0} \leq x_{1} \leq \cdots \leq x_{i} \leq \cdots \leq x_{N}=b, \quad i=0,1, \cdots, N$ and using the quadrature formula, the integral Equation (1) represent a NSVIEs as:

$$
\phi_{n}(t)=W_{n}(t)+\int_{0}^{t} F(t, \tau) \gamma\left(\tau, \phi_{n}(\tau)\right) \mathrm{d} \tau
$$

where $W_{n}(t)=f_{i}(t)+\sum_{i=0}^{n} u_{i} k_{n i} \gamma\left(t, \phi_{i}(t)\right)$.

To solve the NSVIEs:

$$
\varphi(t)=\int_{0}^{t} F(t, \tau) \gamma(\tau, \varphi(\tau)) \mathrm{d} \tau+F(t)+\sum_{i=0}^{n} u_{i} k_{n i} \gamma(t, \varphi(t)), \quad n=0,1, \cdots, N
$$

where

$$
\begin{aligned}
& \varphi(t)=\left(\phi_{1}(t), \phi_{2}(t), \cdots, \phi_{l}(t)\right)^{\mathrm{T}}, \\
& \gamma(\tau, \varphi(\tau))=\left(\gamma\left(\tau, \varphi_{1}(\tau)\right), \gamma\left(\tau, \varphi_{2}(\tau)\right), \cdots, \gamma\left(\tau, \varphi_{l}(\tau)\right)\right), \\
& F(t)=\left(f_{1}(t), f_{2}(t), \cdots, f_{l}(t)\right)^{\mathrm{T}}
\end{aligned}
$$

and

$$
\begin{aligned}
& F(t, \tau) \gamma(\tau, \varphi(\tau)) \\
& =\left[\begin{array}{cccc}
F_{1,1}(t, \tau) \gamma(\tau, \varphi(\tau)) & F_{1,2}(t, \tau) \gamma(\tau, \varphi(\tau)) & \cdots & F_{1, s}(t, \tau) \gamma(\tau, \varphi(\tau)) \\
F_{2,1}(t, \tau) \gamma(\tau, \varphi(\tau)) & F_{2,2}(t, \tau) \gamma(\tau, \varphi(\tau)) & \cdots & F_{2, s}(t, \tau) \gamma(\tau, \varphi(\tau)) \\
\vdots & \vdots & \ddots & \vdots \\
F_{s, 1}(t, \tau) \gamma(\tau, \varphi(\tau)) & F_{s, 2}(t, \tau) \gamma(\tau, \varphi(\tau)) & \cdots & F_{s, s}(t, \tau) \gamma(\tau, \varphi(\tau))
\end{array}\right]
\end{aligned}
$$

Then, we get

$$
\phi_{n}(t)=W_{n}(t)+\int_{0}^{t} F(t, \tau) \gamma(\tau, \varphi(\tau)) \mathrm{d} \tau, \quad n=0,1, \cdots, N
$$

Now, applying the RKM for solve (15):

Suppose that:

$$
F(t, \tau)=\sum_{s} \psi_{s}(t) \chi_{s}(\tau)
$$

Substituting from (16) into (15),

$$
\begin{aligned}
& \phi_{n}(t)=W_{n}(t)+\int_{0}^{t} \sum_{s} \psi_{s}(t) \chi_{s}(\tau) \gamma(\tau, \varphi(\tau)) \mathrm{d} \tau \\
& \phi_{n}(t)=W_{n}(t)+\sum \psi_{s}(t) \int_{0}^{t} \chi_{s}(\tau) \gamma(\tau, \varphi(\tau)) \mathrm{d} \tau
\end{aligned}
$$

Then, we have,

$$
\phi_{n}(t)=W_{n}(t)+\sum_{s} \psi_{s}(t) z_{s}(t), t>0
$$

where 


$$
z_{s}(t)=\int_{0}^{t} \chi_{s}(\tau) \gamma(\tau, \varphi(\tau)) \mathrm{d} \tau
$$

By derivative (18), we have,

$$
z_{s}^{\prime}(t)=\chi_{s}(\tau) \gamma(\tau, \varphi(\tau)), \quad z_{s}(0)=0
$$

Now, apply the RKM to this system of equations to give,

$$
\tilde{z}\left(v_{p} h\right)=h \sum_{q=0}^{p-1} A_{p q} \chi_{s}\left(v_{q} h\right) \gamma\left(v_{q} h, \tilde{\varphi}\left(v_{q} h\right)\right), \quad p=1,2, \cdots, m
$$

which lead to,

$$
\begin{aligned}
\tilde{\phi}_{n}\left(v_{p} h\right) & =W_{n}\left(v_{p} h\right)+\sum_{s} \tilde{z}_{s}\left(v_{p} h\right) \psi_{s}\left(v_{p} h\right) \\
& =W_{n}\left(v_{p} h\right)+\sum_{s} \psi_{s}\left(v_{p} h\right) h \sum_{q=0}^{p-1} A_{p q} \chi_{s}\left(v_{q} h\right) \gamma\left(v_{q} h, \tilde{\varphi}\left(v_{q} h\right)\right) \\
& =W_{n}\left(v_{p} h\right)+h \sum_{q=0}^{p-1} A_{p q} \gamma\left(v_{q} h, \tilde{\varphi}\left(v_{q} h\right)\right) \sum_{s} \psi_{s}\left(v_{p} h\right) \chi_{s}\left(v_{q} h\right)
\end{aligned}
$$

By using Equation (16) to give,

$$
\tilde{\phi}_{n}\left(v_{p} h\right)=W_{n}\left(v_{p} h\right)+h \sum_{q=0}^{p-1} A_{p q} F\left(v_{p} h, v_{q} h\right) \gamma\left(v_{q} h, \tilde{\varphi}\left(v_{q} h\right)\right), \quad p=1,2, \cdots, m
$$

which is approximate solution for Equation (15).

Now, if $m=4$ consider the Pouzet's derivation, we define:

$$
\begin{gathered}
p_{i, j}(t)=\phi_{i, j-1}(t) \\
q_{i, j}(t)=G_{i, j}\left(t_{j+\frac{1}{2}}\right)+\frac{h}{2} F\left(t_{j+\frac{1}{2}}, t_{j}\right) p_{i, j} \\
r_{i, j}(t)=G_{i, j}\left(t_{j+\frac{1}{2}}\right)+\frac{h}{2} F\left(t_{j+\frac{1}{2}}, t_{j+\frac{1}{2}}\right) q_{i, j} \\
s_{i, j}(t)=G_{i, j}\left(t_{j+1}\right)+h F\left(t_{j+1}, t_{j+1}\right) r_{i, j} \\
\phi_{i, j}(t)=G_{i, j}\left(t_{j+1}\right)+\frac{h}{6}\left[F\left(t_{j+1}, t_{j}\right) p_{i, j}+2 F\left(t_{j+1}, t_{j+\frac{1}{2}}\right)\left[q_{i, j}+r_{i, j}\right]+F\left(t_{j+1}, t_{j+1}\right) s_{i, j}\right]
\end{gathered}
$$

The function $\phi_{i, j}(t)$ is unknown function, such that

$$
G_{i, j}(t)=W_{i}(t)+\frac{h}{6} \sum_{n=0}^{j-1}\left[F\left(t, t_{n}\right) p_{i, n}+2 F\left(t, t_{n+\frac{1}{2}}\right)\left[q_{i, n}+r_{i, n}\right]+F\left(t, t_{n+1}\right) s_{i, n}\right]
$$

where $G_{i, 0}(t)=W_{i}(t)$, where $j=2,3, \cdots, N-1$.

when $j=1$, Equation (10) becomes:

$$
\phi_{i, 1}(t)=G_{i, 1}\left(t_{2}\right)+\frac{h}{6}\left[F\left(t_{2}, t_{1}\right) p_{i, 1}+2 F\left(t_{2}, t_{1+\frac{1}{2}}\right)\left[q_{i, 1}+r_{i, 1}\right]+F\left(t_{2}, t_{2}\right) s_{i, 1}\right]
$$

such that $G_{i, 1}=W\left(t_{2}\right)$ and

$$
p_{i, 1}(t)=W_{i}\left(t_{1}\right)
$$




$$
\begin{aligned}
& q_{i, 1}(t)=G_{i, 1}\left(t_{1+\frac{1}{2}}\right)+\frac{h}{2} F\left(t_{1+\frac{1}{2}}, t_{1}\right) p_{i, 1} \\
& r_{i, 1}(t)=G_{i, 1}\left(t_{1+\frac{1}{2}}\right)+\frac{h}{2} F\left(t_{1+\frac{1}{2}}, t_{1+\frac{1}{2}}\right) q_{i, 1} \\
& s_{i, 1}(t)=G_{i, 1}\left(t_{2}\right)+h F\left(t_{2}, t_{2}\right) r_{i, 1}
\end{aligned}
$$

Since the function $\phi_{i, j}(t)=\phi_{i, j}$ is the approximate solution at $\left(x_{i}, t_{j}\right)$ for Equation (1).

\subsection{BBM}

In this section, we use the BBM for solving the NF-VIE of the second kind. The interval $[a, b]$ is divided into steps of width $h, t_{j}=j h, j=0,1, \cdots, n$ and $h=(b-a) / n$. the approximate solution of $\phi_{i}(t)$ will be define at mesh-points $t_{j}$ and denoted by $\phi_{i j}, j=0,1, \cdots, n$ such as $\phi_{i j}$ is an approximation to $\phi_{i}\left(t_{j}\right)$.

To solve the NSVIEs:

$$
\varphi(t)=F(t)+\lambda \sum_{j=0}^{i} u_{j} K_{n j} \gamma(t, \phi(t))+\lambda \int_{0}^{t} F(t, \tau) \gamma(\tau, \varphi(\tau)) \mathrm{d} \tau, \quad n=0,1, \cdots, N
$$

where

$$
\begin{aligned}
& \varphi(t)=\left(\phi_{1}(t), \phi_{2}(t), \cdots, \phi_{l}(t)\right)^{\mathrm{T}}, \\
& \gamma(\tau, \varphi(\tau))=\left(\gamma\left(\tau, \varphi_{1}(\tau)\right), \gamma\left(\tau, \varphi_{2}(\tau)\right), \cdots, \gamma\left(\tau, \varphi_{l}(\tau)\right)\right), \\
& F(t)=\left(f_{1}(t), f_{2}(t), \cdots, f_{l}(t)\right)^{\mathrm{T}}
\end{aligned}
$$

and

$$
\begin{aligned}
& F(t, \tau) \gamma(\tau, \varphi(\tau)) \\
& =\left[\begin{array}{cccc}
F_{1,1}(t, \tau) \gamma(\tau, \varphi(\tau)) & F_{1,2}(t, \tau) \gamma(\tau, \varphi(\tau)) & \cdots & F_{1, s}(t, \tau) \gamma(\tau, \varphi(\tau)) \\
F_{2,1}(t, \tau) \gamma(\tau, \varphi(\tau)) & F_{2,2}(t, \tau) \gamma(\tau, \varphi(\tau)) & \cdots & F_{2, s}(t, \tau) \gamma(\tau, \varphi(\tau)) \\
\vdots & \vdots & \ddots & \vdots \\
F_{s, 1}(t, \tau) \gamma(\tau, \varphi(\tau)) & F_{s, 2}(t, \tau) \gamma(\tau, \varphi(\tau)) & \cdots & F_{s, s}(t, \tau) \gamma(\tau, \varphi(\tau))
\end{array}\right]
\end{aligned}
$$

Then, we get

$$
\phi_{n}(t)=W_{n}(t)+\lambda \int_{0}^{t} F(t, \tau) \gamma(\tau, \varphi(\tau)) \mathrm{d} \tau, \quad n=0,1, \cdots, N
$$

Rewrite Equation (23) as follows:

$$
\begin{gathered}
\phi_{i}\left(t_{k}\right)=W_{i}\left(t_{k}\right)+\lambda \int_{0}^{t_{p m}} F_{i, s}\left(t_{k}, \tau\right) \gamma(\tau, \varphi(\tau)) \mathrm{d} \tau+\lambda \int_{t_{p m}}^{t_{n}} F_{i, s}\left(t_{k}, \tau\right) \gamma(\tau, \varphi(\tau)) \mathrm{d} \tau \\
\phi_{i}\left(t_{k}\right)=f_{i}\left(t_{k}\right)+\lambda \sum_{n=0}^{i} u_{n} k_{i n} \gamma\left(t_{k}, \phi_{n}\left(t_{k}\right)\right)+\lambda \int_{0}^{t_{p m}} F_{i, s}\left(t_{k}, \tau\right) \gamma(\tau, \varphi(\tau)) \mathrm{d} \tau \\
+\lambda \int_{t_{p m}}^{t_{n}} F_{i, s}\left(t_{k}, \tau\right) \gamma(\tau, \varphi(\tau)) \mathrm{d} \tau
\end{gathered}
$$


where $s=1,2, \cdots$.

If the values $\phi_{i 0}, \phi_{i 1}, \cdots, \phi_{i, p m}$ are known, then the first integral can be approximated by standard quadrature methods, and the second integral is obtain by a quadrature rule using values of the integrand at $\tau=t_{p m}, t_{p m+1}, \cdots, t_{p(m+1)}$.

Since the values of $\phi_{i}$ at these points are unknown, we have a system of $l_{p}$ nonlinear equations by applied the BBM:

$$
\begin{aligned}
\phi_{i k}= & f_{i}\left(t_{k}\right)+\lambda \sum_{n=0}^{i} u_{n} K_{i n} \gamma\left(t_{k}, \phi_{n}\left(t_{k}\right)\right) \\
& +\lambda\left[h \sum_{j=0}^{m p} v_{k j} F_{i, s}\left(t_{k}, \tau_{j}\right)\left(\gamma\left(\tau_{j}, \phi_{i j}\right), \cdots, \gamma\left(\tau_{j}, \phi_{i j}\right)\right)\right] \\
& +\lambda\left[h \sum_{j=m p}^{(m+1) p} v_{k j}^{*} F_{i, s}\left(t_{k}, \tau_{m p+j}\right)\left(\gamma\left(\tau_{m p+j}, \phi_{1, m p+j}\right), \cdots, \gamma\left(\tau_{m p+j}, \phi_{1, m p+j}\right)\right)\right]
\end{aligned}
$$

For $n=m p+1, m p+2, \cdots,(m+1) p, \quad m=0,1, \cdots,(N-1)$, where $v_{k j}, v_{k j}^{*}$ depend on the quadrature rule used.

Now, for the Modified method of two Blocks we take $p=2$, this integration over $\left[0, t_{2 m}\right]$ can be accomplished by Simpson's rule, and the integral over $\left[t_{2 m}, t_{n}\right]$ by using a quadratic interpolation of the integrand at the point $t_{2 m}, t_{2 m+1}, t_{2 m+2}$, then Equation (23) becomes:

$$
\phi_{i, 2 m+1}=W_{i}\left(t_{2 m+1}\right)+\lambda \int_{0}^{(2 m+1) h} F_{i, s}\left(t_{2 m+1}, \tau\right) \gamma(\tau, \varphi(\tau)) \mathrm{d} \tau
$$

and

$$
\begin{aligned}
& \phi_{i, 2 m+2} \\
& =W_{i}\left(t_{2 m+2}\right)+\lambda \int_{0}^{(2 m+2) h} F_{i, s}\left(t_{2 m+2}, \tau\right) \gamma(\tau, \varphi(\tau)) \mathrm{d} \tau
\end{aligned}
$$

where $i=1,2, \cdots, l ; m=0,1, \cdots$.

Therefore, by Equation (25) the approximate solution is computed by:

$$
\begin{aligned}
\phi_{i, 2 m+1}= & W_{i}\left(t_{2 m+1}\right)+\lambda\left[\frac{h}{3} \sum_{j=0}^{2 m} v_{j} F_{i, s}\left(t_{2 m+1}, \tau_{j}\right)\left(\gamma\left(\tau_{j}, \phi_{1 j}\right), \cdots, \gamma\left(\tau_{j}, \phi_{1 j}\right)\right)\right] \\
& +\lambda\left[\frac { h } { 1 2 } \left[5 F_{i, s}\left(t_{2 m+1}, \tau_{2 m}\right)\left(\gamma\left(\tau_{2 m}, \phi_{1,2 m}\right), \cdots, \gamma\left(\tau_{2 m}, \phi_{1,2 m}\right)\right)\right.\right. \\
& +8 F_{i, s}\left(t_{2 m+1}, \tau_{2 m+1}\right)\left(\gamma\left(\tau_{2 m+1}, \phi_{1,2 m+1}\right), \cdots, \gamma\left(\tau_{2 m+1}, \phi_{1,2 m+1}\right)\right) \\
& \left.\left.-F\left(t_{2 m+1}, \tau_{2 m+2}\right)\left(\gamma\left(\tau_{2 m+2}, \phi_{1,2 m+2}\right), \cdots, \gamma\left(\tau_{2 m+2}, \phi_{1,2 m+2}\right)\right)\right]\right] \\
\phi_{i, 2 m+2} & W_{i}\left(t_{2 m+2}\right)+\lambda \frac{h}{3} \sum_{j=0}^{2 m+2} v_{j}^{\prime} F_{i, s}\left(t_{2 m+2}, \tau_{j}\right)\left(\gamma\left(\tau_{j}, \phi_{1 j}\right), \cdots, \gamma\left(\tau_{j}, \phi_{1 j}\right)\right)
\end{aligned}
$$

where $u_{i, 0}=g_{i}\left(t_{0}\right)$.

Thus, replace the second term in Equation (28) by using integration formulas, then we get: 


$$
\begin{aligned}
\phi_{i, 2 m+1}= & W_{i}\left(t_{2 m+1}\right)+\lambda\left[\frac{h}{3} \sum_{j=0}^{2 m} v_{j} F_{i, s}\left(t_{2 m+1}, \tau_{j}\right)\left(\gamma\left(\tau_{j}, \phi_{1 j}\right), \cdots, \gamma\left(\tau_{j}, \phi_{l j}\right)\right)\right] \\
+ & \lambda\left[\frac { h } { 6 } \left[F_{i, s}\left(t_{2 m+1}, \tau_{2 m}\right)\left(\gamma\left(\tau_{2 m}, \phi_{1,2 m}\right), \cdots, \gamma\left(\tau_{2 m}, \phi_{1,2 m}\right)\right)\right.\right. \\
& +4 F_{i, s}\left(t_{2 m+1}, \tau_{2 m+\frac{1}{2}}\right)\left(\left(\frac{3}{8} \phi_{1,2 m}+\frac{3}{4} \phi_{1,2 m+1}-\frac{1}{8} \phi_{1,2 m+2}\right), \cdots,\right. \\
& \left.\left(\frac{3}{8} \phi_{1,2 m}+\frac{3}{4} \phi_{l, 2 m+1}-\frac{1}{8} \phi_{l, 2 m+2}\right)\right) \\
+ & \left.\left.F_{l, s}\left(t_{2 m+1}, \tau_{2 m+1}\right)\left(\gamma\left(\tau_{2 m+1}, \phi_{1,2 m+1}\right), \cdots, \gamma\left(\tau_{2 m+1}, \phi_{l, 2 m+1}\right)\right)\right]\right] \\
\phi_{i, 2 m+2} & W_{i}\left(t_{2 m+2}\right)+\lambda\left[\frac{h}{3} F_{i, s}\left(t_{2 m+2}, \tau_{0}\right)\left(\gamma\left(\tau_{0}, \phi_{10}\right), \cdots, \gamma\left(\tau_{0}, \phi_{10}\right)\right)\right. \\
& +4 F_{i, s}\left(t_{2 m+2}, \tau_{1}\right)\left(\gamma\left(\tau_{1}, \phi_{11}\right), \cdots, \gamma\left(\tau_{1}, \phi_{11}\right)\right)+\cdots \\
& \left.+F_{i, s}\left(t_{2 m+2}, \tau_{2 m+2}\right)\left(u_{1,2 m+2}, \cdots, u_{l, 2 m+2}\right)\right]
\end{aligned}
$$

where

$$
\begin{aligned}
& v_{0}=v_{2 m}=1, \quad v_{j}=3-(-1)^{j}, \quad j=1,2, \cdots, 2 m-1 \\
& v_{0}^{\prime}=v_{2 m+2}^{\prime}, \quad v_{j}^{\prime}=3-(-1)^{j}, j=1,2, \cdots, 2 m+1
\end{aligned}
$$

Finally, we construct $2 l$ nonlinear equations from (30) and (31) to find the unknown functions $\phi_{i, 2 m+1}, \phi_{i, 2 m+2}$. The resulting system is solved by using modified Newton-Raphson method.

\section{Numerical Examples}

We solve two examples by RKM and BBMat $N=20,50, T=0.01,0.1,0.3$, $\lambda=1$ and $\mu=1$.

In Tables 1-6: $\phi_{\text {Exact }} \rightarrow$ Exact solution, $\phi_{R . K} \rightarrow$ approximate solution of RKM, $E_{R . K .} \rightarrow$ the absolute error of RKM, $\phi_{B . B .} \rightarrow$ approximate solution of BBM, $E_{B . B .} \rightarrow$ the absolute error of BBM.

Example 1

Consider: $\phi(x, t)=x t-\frac{x^{2} t^{2}}{5}-\frac{x^{2} t^{6}}{5}+\int_{0}^{1} x^{2} y^{2} \phi^{2}(y, t) \mathrm{d} y+\int_{0}^{t} t \tau \phi^{2}(x, \tau) \mathrm{d} \tau$

Exact solution $\phi(x, t)=x t$

Case 1: $N=20$

Table 1. The values of exact, approximate solutions, and errors by using RKM and BBM at $T=0.01, N=20$.

\begin{tabular}{cccccc}
\hline$x$ & $\phi_{\text {Exact }}$ & $\phi_{\text {R.K. }}$ & $E_{\text {R.K. }}$ & $\phi_{\text {B.B. }}$ & $E_{\text {B.B. }}$ \\
\hline 0 & 0 & 0 & 0 & 0 & 0 \\
0.1 & 0.0001000 & 0.0001499 & $4.9997 \times 10^{-5}$ & 0.0000999 & $2.4167 \times 10^{-10}$ \\
0.2 & 0.0004000 & 0.0004999 & $9.9978 \times 10^{-5}$ & 0.0003999 & $3.853 \times 10^{-9}$ \\
0.3 & 0.0009000 & 0.0010499 & $1.4992 \times 10^{-4}$ & 0.0008999 & $1.9247 \times 10^{-8}$ \\
\hline
\end{tabular}




\section{Continued}

\begin{tabular}{cccccc}
\hline 0.4 & 0.0016000 & 0.0017998 & $1.9980 \times 10^{-4}$ & 0.0015994 & $5.8590 \times 10^{-8}$ \\
0.5 & 0.0025000 & 0.0027496 & $2.4960 \times 10^{-4}$ & 0.0024998 & $1.3151 \times 10^{-7}$ \\
0.6 & 0.0036000 & 0.0038993 & $2.9932 \times 10^{-4}$ & 0.0035997 & $2.2922 \times 10^{-7}$ \\
0.7 & 0.0049000 & 0.0052490 & $3.4901 \times 10^{-4}$ & 0.0048997 & $2.9202 \times 10^{-7}$ \\
0.8 & 0.0064000 & 0.0067988 & $3.9884 \times 10^{-4}$ & 0.0063998 & $1.5103 \times 10^{-7}$ \\
0.9 & 0.0081000 & 0.0085491 & $4.4918 \times 10^{-4}$ & 0.0081005 & $5.6689 \times 10^{-7}$ \\
1 & 0.0100000 & 0.0104981 & $4.9815 \times 10^{-4}$ & 0.0100000 & $8.334 \times 10^{-8}$ \\
\hline
\end{tabular}

Table 2. The values of exact, approximate solutions, and errors by using RKM and BBM at $T=0.1, N=20$.

\begin{tabular}{cccccc}
\hline$x$ & $\phi_{\text {Exact }}$ & $\phi_{\text {R.K. }}$ & $E_{\text {R.K. }}$ & $\phi_{\text {B.B. }}$ & $E_{\text {B.B. }}$ \\
\hline 0 & 0 & 0 & 0 & 0 & 0 \\
0.1 & 0.0010000 & 0.0014997 & $4.9997 \times 10^{-4}$ & 0.0009999 & $2.4161 \times 10^{-8}$ \\
0.2 & 0.0040000 & 0.0049978 & $9.9978 \times 10^{-4}$ & 0.0039996 & $3.853 \times 10^{-7}$ \\
0.3 & 0.0090000 & 0.0104922 & $1.4922 \times 10^{-3}$ & 0.0089980 & $1.9247 \times 10^{-6}$ \\
0.4 & 0.0160000 & 0.0179805 & $1.9805 \times 10^{-3}$ & 0.0159941 & $5.8591 \times 10^{-6}$ \\
0.5 & 0.0250000 & 0.0274608 & $2.4608 \times 10^{-3}$ & 0.0249868 & $1.3152 \times 10^{-5}$ \\
0.6 & 0.0360000 & 0.0389329 & $2.9329 \times 10^{-3}$ & 0.0359770 & $2.2927 \times 10^{-5}$ \\
0.7 & 0.0490000 & 0.0524020 & $3.4020 \times 10^{-3}$ & 0.0489707 & $2.9217 \times 10^{-5}$ \\
0.8 & 0.0640000 & 0.0679851 & $3.8851 \times 10^{-3}$ & 0.0639848 & $1.5121 \times 10^{-5}$ \\
0.9 & 0.0810000 & 0.0854199 & $4.4199 \times 10^{-3}$ & 0.0810568 & $5.682 \times 10^{-5}$ \\
1 & 0.1000000 & 0.1048178 & $4.8178 \times 10^{-3}$ & 0.1000083 & $8.35 \times 10^{-6}$ \\
\hline
\end{tabular}

Table 3. The values of exact, approximate solutions, and errors by using RKM and BBM at $T=0.3, N=20$.

\begin{tabular}{cccccc}
\hline$X$ & $\phi_{\text {Exact }}$ & $\phi_{\text {R.K. }}$ & $E_{\text {R.K. }}$ & $\phi_{\text {B.B. }}$ & $E_{\text {B.B. }}$ \\
\hline 0 & 0 & 0 & 0 & 0 & 0 \\
0.1 & 0.0030000 & 0.0049753 & $1.4975 \times 10^{-3}$ & 0.0029997 & $2.1745 \times 10^{-7}$ \\
0.2 & 0.0120000 & 0.0014980 & $2.9804 \times 10^{-3}$ & 0.0119965 & $3.4685 \times 10^{-6}$ \\
0.3 & 0.0270000 & 0.0314308 & $4.4308 \times 10^{-3}$ & 0.0269826 & $1.7322 \times 10^{-5}$ \\
0.4 & 0.0480000 & 0.0538289 & $5.8289 \times 10^{-3}$ & 0.0479472 & $5.2734 \times 10^{-5}$ \\
0.5 & 0.0750000 & 0.0821593 & $7.1593 \times 10^{-3}$ & 0.0748816 & $1.1838 \times 10^{-4}$ \\
0.6 & 0.1080000 & 0.1164275 & $8.4275 \times 10^{-3}$ & 0.1077935 & $2.0644 \times 10^{-4}$ \\
0.7 & 0.1470000 & 0.1566904 & $9.6904 \times 10^{-3}$ & 0.1467367 & $2.6328 \times 10^{-4}$ \\
0.8 & 0.1920000 & 0.2031120 & $1.1112 \times 10^{-2}$ & 0.1918634 & $1.3654 \times 10^{-4}$ \\
0.9 & 0.2430000 & 0.2560541 & $1.3054 \times 10^{-2}$ & 0.2435136 & $5.1366 \times 10^{-4}$ \\
1 & 0.3000000 & 0.3138329 & $1.3832 \times 10^{-2}$ & 0.3000744 & $7.4446 \times 10^{-5}$ \\
\hline & & & & & \\
\hline
\end{tabular}


Case 2: $\quad N=50$

Table 4. The values of exact, approximate solutions, and errors by using RKM and BBM at $T=0.01, N=50$.

\begin{tabular}{cccccc}
\hline$X$ & $\phi_{\text {Exact }}$ & $\phi_{R . K .}$ & $E_{R . K .}$ & $\phi_{\text {B.B. }}$ & $E_{\text {B.B. }}$ \\
\hline 0 & 0 & 0 & 0 & 0 & 0 \\
0.1 & 0.0001000 & 0.0001199 & $1.9999 \times 10^{-5}$ & 0.0000999 & $9.867 \times 10^{-11}$ \\
0.2 & 0.0004000 & 0.0004399 & $3.9991 \times 10^{-5}$ & 0.0003999 & $1.5735 \times 10^{-9}$ \\
0.3 & 0.0009000 & 0.0009599 & $5.9969 \times 10^{-5}$ & 0.0008999 & $7.8606 \times 10^{-9}$ \\
0.4 & 0.0016000 & 0.0016799 & $7.9923 \times 10^{-5}$ & 0.0015999 & $2.3949 \times 10^{-8}$ \\
0.5 & 0.0025000 & 0.0025998 & $9.9844 \times 10^{-5}$ & 0.0024999 & $5.3855 \times 10^{-8}$ \\
0.6 & 0.0036000 & 0.0037197 & $1.1973 \times 10^{-4}$ & 0.0035999 & $9.4282 \times 10^{-8}$ \\
0.7 & 0.0049000 & 0.0050396 & $1.3960 \times 10^{-4}$ & 0.0048998 & $1.2160 \times 10^{-7}$ \\
0.8 & 0.0064000 & 0.0065595 & $1.5952 \times 10^{-4}$ & 0.0063999 & $6.8595 \times 10^{-8}$ \\
0.9 & 0.0081000 & 0.0082796 & $1.7964 \times 10^{-4}$ & 0.0081002 & $2.1360 \times 10^{-7}$ \\
1 & 0.0100000 & 0.0101992 & $1.9922 \times 10^{-4}$ & 0.0100000 & $1.333 \times 10^{-8}$ \\
\hline
\end{tabular}

Table 5. The values of exact, approximate solutions, and errors by using RKM and BBM at $T=0.1, N=50$.

\begin{tabular}{cccccc}
\hline$x$ & $\phi_{\text {Exact }}$ & $\phi_{\text {R.K. }}$ & $E_{\text {R.K. }}$ & $\phi_{\text {B.B. }}$ & $E_{\text {B.B. }}$ \\
\hline 0 & 0 & 0 & 0 & 0 & 0 \\
0.1 & 0.0010000 & 0.0011999 & $1.9990 \times 10^{-4}$ & 0.0009999 & $9.8647 \times 10^{-9}$ \\
0.2 & 0.0040000 & 0.0043991 & $3.9917 \times 10^{-4}$ & 0.0039998 & $1.5735 \times 10^{-7}$ \\
0.3 & 0.0090000 & 0.0095968 & $5.9698 \times 10^{-4}$ & 0.0089992 & $7.8607 \times 10^{-7}$ \\
0.4 & 0.0160000 & 0.0167923 & $7.9237 \times 10^{-4}$ & 0.0159976 & $2.3948 \times 10^{-6}$ \\
0.5 & 0.0250000 & 0.0259844 & $9.8448 \times 10^{-4}$ & 0.0249946 & $5.3855 \times 10^{-6}$ \\
0.6 & 0.0360000 & 0.0371732 & $1.1732 \times 10^{-3}$ & 0.0359905 & $9.4288 \times 10^{-6}$ \\
0.7 & 0.0490000 & 0.0503606 & $1.3606 \times 10^{-3}$ & 0.0489878 & $1.2163 \times 10^{-5}$ \\
0.8 & 0.0640000 & 0.0655530 & $1.5530 \times 10^{-3}$ & 0.0639931 & $6.8630 \times 10^{-6}$ \\
0.9 & 0.0810000 & 0.0827654 & $1.7654 \times 10^{-3}$ & 0.0810213 & $2.1380 \times 10^{-5}$ \\
1 & 0.1000000 & 0.1019246 & $1.9246 \times 10^{-3}$ & 0.1000013 & $1.3344 \times 10^{-6}$ \\
\hline
\end{tabular}

Table 6. The values of exact, approximate solutions, and errors by using RKM and BBM at $T=0.3, N=50$.

\begin{tabular}{cccccc}
\hline$X$ & $\phi_{\text {Exact }}$ & $\phi_{\text {R.K. }}$ & $E_{\text {R.K. }}$ & $\phi_{\text {B.B. }}$ & $E_{\text {B.B. }}$ \\
\hline 0 & 0 & 0 & 0 & 0 & 0 \\
0.1 & 0.0030000 & 0.0035991 & $5.9912 \times 10^{-4}$ & 0.0029999 & $8.8781 \times 10^{-8}$ \\
0.2 & 0.0120000 & 0.0013192 & $1.1925 \times 10^{-3}$ & 0.0119985 & $1.4161 \times 10^{-6}$ \\
0.3 & 0.0270000 & 0.0287733 & $1.7733 \times 10^{-3}$ & 0.0269929 & $7.0746 \times 10^{-6}$ \\
0.4 & 0.0480000 & 0.0503338 & $2.3338 \times 10^{-3}$ & 0.0479784 & $2.1553 \times 10^{-5}$ \\
0.5 & 0.0750000 & 0.0778692 & $2.8692 \times 10^{-3}$ & 0.0749515 & $4.8471 \times 10^{-5}$ \\
\hline
\end{tabular}




\begin{tabular}{cccccc} 
Continued & \multicolumn{3}{l}{} \\
\hline 0.6 & 0.1080000 & 0.1113838 & $3.3838 \times 10^{-3}$ & 0.1079151 & $8.4876 \times 10^{-5}$ \\
0.7 & 0.1470000 & 0.1509044 & $3.9044 \times 10^{-3}$ & 0.1468904 & $1.0951 \times 10^{-4}$ \\
0.8 & 0.1920000 & 0.1965019 & $4.5019 \times 10^{-3}$ & 0.1919381 & $6.1870 \times 10^{-5}$ \\
0.9 & 0.2430000 & 0.2483267 & $5.3267 \times 10^{-3}$ & 0.2431926 & $1.9269 \times 10^{-4}$ \\
1 & 0.3000000 & 0.3057405 & $5.7405 \times 10^{-3}$ & 0.3000111 & $1.1596 \times 10^{-5}$ \\
\hline
\end{tabular}

\section{Conclusions}

From the previous discussions we conclude the following:

1) As $N$ is increasing the errors are decreasing.

2) As $x$ and $t$ are increasing in $[0,1] \times[0,1]$, the errors due to RKM and BBM are also increasing.

3) The errors due to the BBM are less than the errors due to RKM (i.e. BBM the better than RKM to solve NF-VIE with continuous kernel).

\section{Conflicts of Interest}

The authors declare no conflicts of interest regarding the publication of this paper.

\section{References}

[1] Linz, P. (1985) Analytic and Numerical Methods for Volterra Equations. SIAM, Philadelphia. https://doi.org/10.1137/1.9781611970852

[2] Mirzaee, F. and Rafei, Z. (2011) The Block by Block Method for the Numerical Solution of the Nonlinear Two-Dimensional Volterra Integral Equations. Journal of king Saud University-Science, 23, 191-195. https://doi.org/10.1016/j.jksus.2010.07.008

[3] Maleknejad, K. and Shahrezaee, M. (2004) Using Runge-Kutta Method for Numerical Solution of the System of Volterra Integral Equation. Applied Mathematics and Computation, 149, 399-410. https://doi.org/10.1016/S0096-3003(03)00148-6

[4] Maleknejad, K. and Ostadi, A. (2017) Numerical Solution of System of Volterra Integral Equations with Weakly Singular Kernels and Its Convergence Analysis. Applied Numerical Mathematics, 115, 82-98. https://doi.org/10.1016/j.apnum.2016.12.005

[5] Armand, A. and Gouyandch, Z. (2014) Numerical Solution of the System of Volterra Integral Equations of the First Kind. IJIM, 6, 9.

[6] Biazar, J. and Babolian, E. (2003) Solution of a System of Volterra Integral Equations of the First Kind by Adomian Method. Applied Mathematics and Computation, 139, 249-258. https://doi.org/10.1016/S0096-3003(02)00173-X

[7] Yaghouti, M.R. (2012) A Numerical Method for Solving a System of Volterra Integral Equations. WAP, 2, 18-33.

[8] Katani, R. and Shahmorad, S. (2010) Block by Block Method for the Systems of Nonlinear Volterra Integral Equations. Applied Mathematical Modelling, 34, 400-406. https://doi.org/10.1016/j.apm.2009.04.013

[9] Al-Wagdani, M.M. (2016) On Some Numerical Treatments for Solving Volterra Integral Equation and System of Volterra Integral Equations. Taif University, Taif. 